\title{
Efeu in der pädiatrischen Praxis
}

\section{Anwendung von EA $575^{\circledR}$ in der Therapie der akuten Bronchitis bei Schulkindern}

\author{
Christopher Lang, Christiane Staiger, Tankred Wegener
}

\begin{abstract}
Zusammenfassung
In einer nicht-interventionellen Studie wurden die Wirksamkeit und Verträglichkeit 5 unterschiedlicher Darreichungsformen mit dem Efeu-Spezialextrakt EA $575^{\circledR}$ bei über 1000 Schulkindern (Alter 6-12 Jahre) mit akuter Bronchitis untersucht. Die Wirkung wurde durch eine ärztliche Befundbewertung, durch eine Bewertung der Patienten mittels Abfrage typischer Symptome sowie mittels eines Tagebuches erfasst. Bei allen Befunden und Symptomen kam es im Verlauf der 7-tägigen Therapie zu einer deutlichen Verbesserung. Zudem zeigten sich eine sehr gute Verträglichkeit und eine hohe Compliance. Alle 5 untersuchten EA $575^{\circledR}$-Darreichungsformen stellen damit eine wirksame und sichere Therapie der akuten Bronchitis im untersuchten Kollektiv der Schulkinder dar und bestätigen somit die langjährige Erfahrung mit EA $575^{\circledR}$.
\end{abstract}

Husten zählt über alle Altersklassen hinweg zu den am häufigsten vorgebrachten Beschwerden in der allgemeinärztlichen Praxis mit einer erheblichen sozioökonomischen Bedeutung. Vor allem unter den Kindern gilt Husten zusammen mit Fieber als das häufigste Symptom und trägt im Schulalter maßgeblich zum Auftreten von Fehltagen bei [2, 3, 14]. Beispielsweise konnte in einer Beobachtungsstudie festgestellt werden, dass im Zeitraum eines Jahres 27,6\% der Kinder aufgrund respiratorischer Symptome dem Unterricht für 1-20 Tage fernbleiben mussten [3]. Ursächlich für den Husten in dieser Altersgruppe ist sehr häufig eine akute Bronchitis durch eine meist virale Infektion der Atemwege. Als sekundärer Reinigungsmechanismus und Schutzreflex der Lunge dient der Husten dazu, Fremdkörper, übermäßigen Schleim sowie Patho- gene aus dem Atmungssystem zu entfernen. Er kann durch entzündliche, chemische, physikalische oder mechanische Reizungen der Schleimhaut der oberen und unteren Atemwege ausgelöst werden [2]. Von der akuten Bronchitis abzugrenzen ist die chronische Bronchitis. Von dieser spricht man nach einer WHO-Definition dann, wenn bei einem Patienten an mindestens 3 aufeinanderfolgenden Monaten während 2 Jahren Husten mit oder ohne Auswurf bestand.

In einer kürzlich veröffentlichten Studie konnte gezeigt werden, dass das Symptom Husten nicht nur für die Patienten selbst, sondern auch für deren Eltern eine beträchtliche Belastung darstellt [10]. Daher ist eine wirksame und verträgliche Therapie der Symptomatik für die Patienten sowie deren Eltern von hoher Bedeutung. Seit 65 Jahren und in bis heute mehr als 300 Mio. Anwendungen bewähren sich pflanzliche Arzneimittel mit dem Efeu-Spezialextrakt EA $575^{\circledR}$ (Prospan ${ }^{\circledR}$, Engelhard Arzneimittel) bei Husten [1, 5, $12,15]$. Sie gelten als Alternative zu synthetischen Arzneistoffen zur Besserung der Beschwerden bei chronisch-entzündlichen Bronchialerkrankungen und akuten Entzündungen der Atemwege mit der Begleiterscheinung Husten. Mittlerweile liegen zu EA $575^{\circledR}$ klinische Daten von mehr als 65000 Patienten vor [8]. In der vorliegenden nicht-interventionellen Studie sollte ein besonderes Augenmerk auf Kinder im Schulalter gelegt werden.

\section{Studienziel}

Die nicht-interventionelle Studie (NIS) diente der Erfassung der Wirksamkeit, Compliance und Verträglichkeit der Therapie von Schulkindern (Alter: 6-12 Jah-

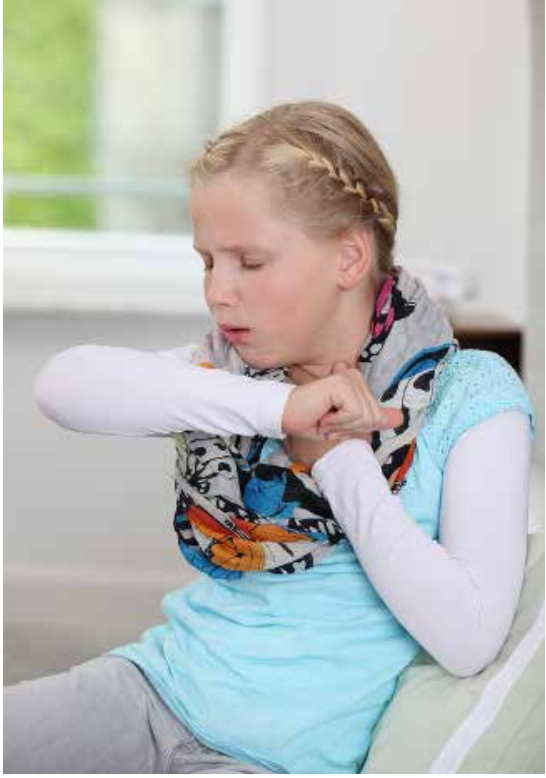

Phytotherapeutisch gut zugänglich: akute Bronchitis. @ Engelhard Arzneimitte

re) mit akuter Bronchitis mit Prospan ${ }^{\circledR}$. Alle 5 im Handel befindlichen Darreichungsformen - Hustensaft, Hustentropfen, Hustenliquid, Husten-Brausetabletten, Husten-Lutschpastillen - enthalten den Efeublätter-Spezialextrakt EA $575^{\circledR}$ (DEV 5-7,5:1, Auszugsmittel Ethanol 30\% $[\mathrm{m} / \mathrm{m}])$ und wurden in die NIS einbezogen.

\section{Methode}

Insgesamt nahmen 201 Ärzte, überwiegend Pädiater, an der NIS im Zeitraum der Erkältungssaison 2014/2015 teil. Im Rahmen ihrer täglichen Praxis wurden Kinder mit der Diagnose akute Bronchitis zur Teilnahme an der Studie eingeladen. Ein Einschluss in die Studie war nur nach mündlicher und schriftlich dokumentierter Aufklärung der Eltern und Patienten und nach Unterzeichnung einer Ein- 
verständniserklärung möglich. Die Therapieentscheidung des Arztes und der Einschluss der Patienten erfolgten unbeeinflusst und unabhängig von der Studiensituation. Das bedeutete, dass nur Patienten vom Arzt ausgewählt und eingeschlossen werden durften, die auch im Rahmen der täglichen Praxis mit den EA $575^{\circledR}$-haltigen Arzneimitteln behandelt worden wären.

Bei der Aufnahmeuntersuchung wurden demografische und anamnestische Daten sowie die Aufnahmeindikation und typische Beschwerden im Beobachtungsbogen erfasst. Anschließend wurde eine der fünf EA $575^{\circledR}$-Darreichungsformen vom Arzt verordnet. Nach ca. 1 Woche wurden bei der Abschlussvisite Angaben zur Therapie und zum Symptomverlauf im Beobachtungsbogen dokumentiert. Folgende klinische Befunde wurden durch die Ärzte anhand spezifischer Scores (3- bis 7-stufig) bei beiden Visiten dokumentiert: Hustenanfälle, Auswurf/ Sputum, Dyspnoe während des Hustens, rasselnde Atemgeräusche/Auskultation, Schweregrad der Erkrankung, Hustenqualität/Auskultation. Weiterhin bewerteten die Patienten selbst die folgenden Symptome, die durch den Arzt abgefragt wurden: Hustenintensität, Brustschmerz während des Hustens, Atemnot während des Hustens, hustenbedingte Schlafstörungen, Abhusten von Schleim, Anzahl von Hustenattacken während des Tages. Ebenfalls erfolgte bei der Abschlussvisite die Bewertung des Geschmacks des verordneten Arzneimittels.

Die durch den Arzt klinisch erfassten Symptome Hustenanfälle, Auswurf/Sputum, Dyspnoe und Atemgeräusche dienten zusammen mit der Evaluation der Brustschmerzen beim Husten durch die Patienten als Grundlage für die Berechnung des Bronchitis Severitiy Scores (BSS), der bereits mehrfach in kontrollierten klinischen Studien als validierter Score Anwendung gefunden hat $[6,11]$.

Zusätzlich dokumentierten die Kinder bzw. deren Eltern täglich den Verlauf der Symptome in einem Patiententagebuch (Anzahl Hustenattacken, Hustenintensität, Brustschmerz beim Husten, Atemnot, Abhusten von Schleim, hustenbedingte Schlafstörungen, Häufigkeit des nächtlichen Aufwachens, allgemeine Beschwer- den oder Symptome, Compliance; jeweils 4- bis 5-stufige Scores).

Bei der Abschlussuntersuchung wurde die Compliance durch den Arzt erfragt sowie anhand der Daten aus dem Patiententagebuch überprüft. Des Weiteren wurde die allgemeine Wirksamkeit durch die Ärzte und durch die Patienten bzw. deren Eltern bewertet. Die Verträglichkeit wurde bei der Abschlussvisite durch die Einschätzung der globalen Verträglichkeit sowohl durch den Arzt als auch den Patienten bzw. seine Eltern erfasst. Jede aufgetretene unerwünschte Arzneimittelwirkung (UAW) wurde gemäß der üblichen regulatorischen Vorgehensweise bewertet und berichtet.

Die Auswertung aller dokumentierter Daten erfolgte im intraindividuellen Vergleich mittels deskriptiver statistischer Verfahren (explorativ). Als Drop-out wurden nur Patienten betrachtet, die ohne Angabe von Gründen und ohne Angaben aus einer Folgeuntersuchung nicht wieder in der Praxis erschienen waren und für die keine ausreichenden Daten aus einer Abschlussuntersuchung vorlagen.

Die multizentrische, prospektive NIS wurde gemäß $\S 4$ (23) und $\S 67$ (6) AMG, unter Beachtung der früheren Bekanntmachung des BfArM [4] sowie den Empfehlungen der Gesellschaft für Phytotherapie (GPT) [7, 16, 17] zur Planung, Durchführung und Auswertung von Anwendungsbeobachtungen durchgeführt. Gemäß den regulatorischen Vorgaben wurde die NIS den zuständigen Stellen angezeigt sowie der Ethikkommission der Ärztekammer Westfalen-Lippe und der Medizinischen Fakultät der Westfälischen Wilhelms-Universität Münster vorgelegt und von dieser unter dem Aktenzeichen 2014-366-f-S ohne Bedenken ethischer oder rechtlicher Art befürwortet.

\section{Studienpopulation}

Insgesamt wurden 1088 Schulkinder mit akuter Bronchitis unterschiedlicher Genese in die Studie eingeschlossen. Die Daten von 1066 Patienten (525 Mädchen, 537 Jungen, 4 k.A.), davon 829 mit Patiententagbuch, konnten ausgewertet werden. Darunter befanden sich 8 Kinder ( 7 jünger als 6, eines älter als 12 Jahre), die trotz der Abweichung im zu beobachten- den Alter in die Gesamtauswertung von Wirksamkeit und Verträglichkeit einbezogen wurden. Das mittlere Alter der Kinder betrug 8,5 \pm 0,06 Jahre. Die bisherige Dauer der Beschwerden wurde im Mittel mit 2,29 $\pm 0,05$ Tagen angegeben. Die Behandlung der Patienten erfolgte im Großteil der Fälle mit Saft oder Liquid ( $n=719 ; n=196$ ) gefolgt von Tropfen, Lutschpastillen und Brausetabletten $(n=64 ; n=49 ; n=38)$. Die Dauer der Behandlung betrug im Mittel 6,92 $\pm 0,05$ Tage.

Bei 27,0\% ( $n=288)$ erfolgte eine Begleittherapie der Atemwegserkrankung. Am häufigsten wurden hierbei Nasenspray und -tropfen $(\mathrm{n}=95)$ sowie Antibiotika $(n=28)$ angewendet. Neben der Einschlussindikation gaben $10,0 \%(n=107)$ der Patienten an, an einer Begleitinfektion (meist Otitis, (Rhino)sinusitis) zu leiden. Diese wurden, falls medikamentös therapiert, mehrheitlich antibiotisch $(n=47)$ behandelt. 19,6\% $(n=209)$ der Kinder litten unter einer relevanten Begleiterkrankung. Hierbei wurde Asthma am häufigsten genannt $(n=44)$. Die Behandlung der Begleiterkrankung erfolgte indikationsspezifisch. Bei insgesamt $11,1 \%(n=118)$ der Patienten lag eine allergische Disposition vor.

\section{Ergebnisse}

\section{Wirksamkeit - Bewertung durch die Ärzte}

Die durch den Arzt klinisch erfassten Symptome Hustenanfälle, Auswurf/Sputum, Dyspnoe und Atemgeräusche besserten sich bei allen Darreichungsformen innerhalb der Beobachtungsdauer von einer Woche in einem vergleichbaren Ausmaß jeweils im Mittel um etwa 50\% ( $\triangleright$ Tab. 1). Der BSS zeigte eine deutliche Verbesserung im Mittel von 6,23 auf 1,29 um 79,3\%. Interessant ist hierbei die Tatsache, dass es keinen Unterschied in der relativen Verbesserung zwischen den Gruppen mit (77,9\%) und ohne Begleittherapie (80,2\%) gab ( $\triangleright$ Abb. 1). Auch zwischen den verschiedenen Darreichungsformen konnte kein relevanter Unterschied in der Verbesserung des BSS beobachtet werden. Diese lagen zwischen $74,3 \%$ und $85,0 \%$ ( $\triangleright$ Tab. 2 ) 
Der Schweregrad der Erkrankung (CGI-Scale) besserte sich nach Einschätzung des Arztes im Mittel von 4,32 \pm 0,03 auf $1,79 \pm 0,03$ und damit um 58,6\% auf die entsprechende Einstufung „Grenzfall einer Erkrankung“. Die Zahl der deutlich, schwer und sehr schwer Erkrankten verminderte sich dabei von 431 auf insgesamt 16 , d.h. um $96,3 \%$.

Die Bestimmung der Hustenqualität erfolgte durch die Erhebung auskultatorischer Befunde durch den Arzt. Hier zeigte sich im Verlauf des Therapiezeitraums eine Reduktion des Patientenanteils mit trockenem Husten von 46,2\% auf 10,6\%, für produktiven Husten von 43,2\% auf 15,5\%. Der Anteil von Patienten ohne auskultatorisch auffälligen Befund erhöhte sich dagegen von $8,2 \%$ auf $63,2 \%$.

\section{Wirksamkeit - Bewertung durch Patienten bzw. deren Eltern}

Die subjektiv wahrgenommenen Symptome Hustenintensität, Brustschmerz während des Hustens, Atemnot während des Hustens und hustenbedingte Schlafstörungen wurden bei der Eingangs- und Abschlussuntersuchung vom Arzt bei den Patienten bzw. deren Eltern erfragt. Hierbei konnte je nach Symptomatik im Therapieverlauf eine Verbesserung von 49,9$58,1 \%$ beobachtet werden ( $\triangleright$ Tab. 3 ). Die genannten Symptome wurden darüber hinaus zusätzlich im Patiententagebuch an den Therapietagen 1-6 abgefragt und zeigen im Verlauf eine sehr gute Übereinstimmung mit den im Rahmen der beiden Visiten erhobenen Daten (Intensität: $3,40 \pm 0,03$ auf $1,68 \pm 0,02$; Brustschmerz: $2,72 \pm 0,03$ auf $1,15 \pm 0,02$; Atemnot: 2,63 $\pm 0,04$ auf $1,13 \pm 0,02$; Schlafstörungen: $3,05 \pm 0,03$ auf $1,26 \pm 0,02$ ).

Weiterhin wurden sowohl im Rahmen der ärztlichen Visiten als auch im Patiententagebuch die Problematik des Abhustens von Schleim und die Anzahl der Hustenattacken pro Tag abgefragt. Das Abhusten verbesserte sich im Therapieverlauf von 3,13 auf 1,50. Bei der Bewertung der Anzahl von Hustenattacken lag bei der Aufnahme mit einem Score von im Mittel 2,76 Punkten eine rechnerische Häufigkeit zwischen 15 und 20 Hustenattacken pro Tag vor. Im Behandlungsverlauf verbesserte sich die Häufigkeit auf im

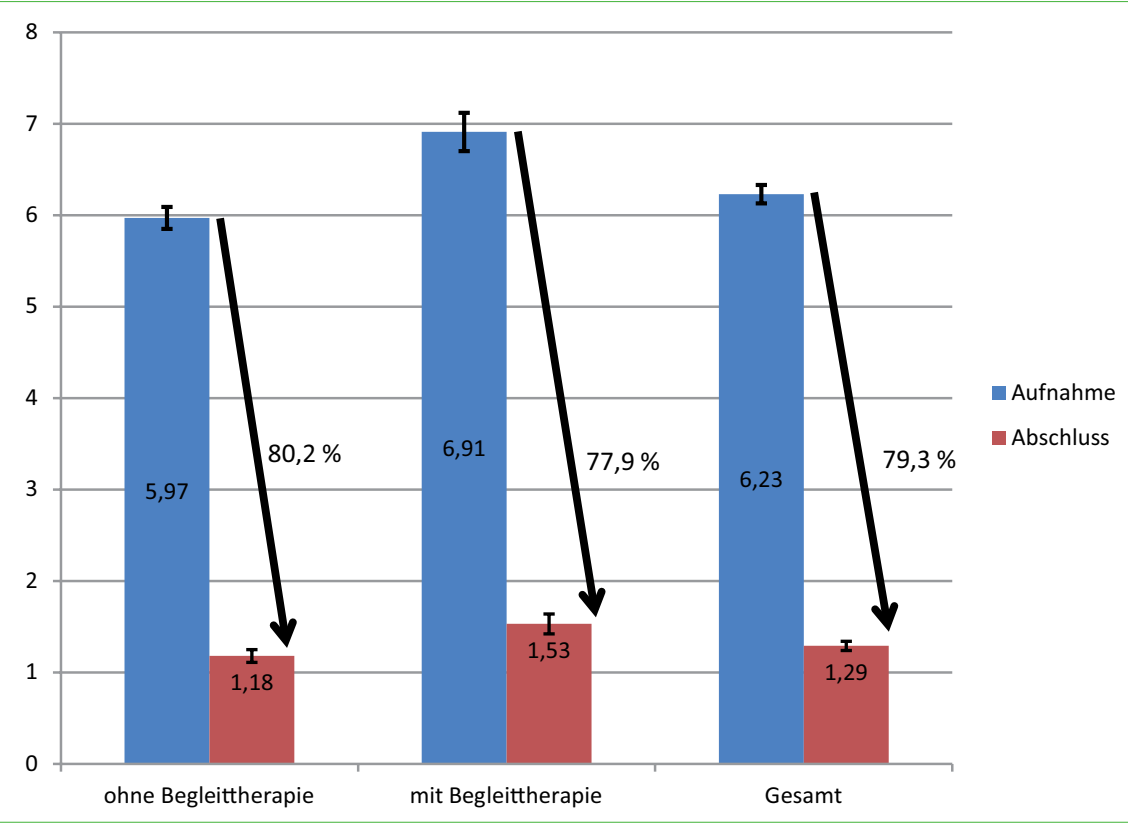

- Abb. 1 Verbesserung des berechneten BSS im Therapieverlauf im Kollektiv der Patienten ohne und mit Begleittherapie sowie im Gesamtkollektiv.

- Tab. 1 Mittlere Symptomausprägung (jeweils 5-stufiger Score) im Urteil des Arztes bei der Aufnahme- und der Abschlussvisite sowie mittlere Veränderung in \%.

\begin{tabular}{|c|c|c|c|c|}
\hline Symptom & Aufnahme & Abschluss & $\begin{array}{l}\text { Veränderung } \\
\text { [absolut] }\end{array}$ & $\begin{array}{l}\text { Veränderung } \\
\text { [\%] }\end{array}$ \\
\hline Hustenanfälle & $3,18 \pm 0,02$ & $1,62 \pm 0,02$ & 1,56 & 49,1 \\
\hline Auswurf/Sputum & $2,58 \pm 0,02$ & $1,38 \pm 0,02$ & 1,20 & 46,6 \\
\hline Dyspnoe & $2,48 \pm 0,03$ & $1,14 \pm 0,02$ & 1,34 & 54,2 \\
\hline Atemgeräusche & $2,61 \pm 0,03$ & $1,25 \pm 0,02$ & 1,36 & 52,1 \\
\hline
\end{tabular}

- Tab. 2 BSS-Score bei Aufnahme und Abschluss für das Gesamtkollektiv und die einzelnen Darreichungsformen (Mittelwerte der Scores und Veränderungen in \%).

\begin{tabular}{l|l|l|l|l|l|l|}
$\begin{array}{l}\text { Symptom/Darrei- } \\
\text { chungsform }\end{array}$ & Tropfen & Saft & Liquid & $\begin{array}{l}\text { Lutsch- } \\
\text { pastillen }\end{array}$ & $\begin{array}{l}\text { Brause- } \\
\text { tabletten }\end{array}$ & gesamt \\
\hline Aufnahme & 7,35 & 6,12 & 6,37 & 5,64 & 6,45 & 6,23 \\
& $\pm 0,45$ & $\pm 0,13$ & $\pm 0,25$ & $\pm 0,48$ & $\pm 0,55$ & $\pm 0,10$ \\
\hline Abschluss & 1,87 & 1,28 & 1,14 & 0,84 & 1,66 & 1,29 \\
\hline Veränderung [\%] & 74,6 & 79,1 & 82,1 & 85,0 & 74,3 & 79,3 \\
\hline
\end{tabular}

- Tab. 3 Mittlere Symptomausprägung aus Befragung der Patienten/deren Eltern durch den Arzt bei der Aufnahme- und der Abschlussvisite und mittlere Veränderung in \%.

\begin{tabular}{|c|c|c|c|c|c|}
\hline Symptom & Score & Aufnahme & Abschluss & $\begin{array}{l}\text { Veränderung } \\
\text { [absolut] }\end{array}$ & $\begin{array}{l}\text { Veränderung } \\
\text { [\%] }\end{array}$ \\
\hline Hustenintensität & 5-stufig & $3,32 \pm 0,02$ & $1,66 \pm 0,02$ & 1,66 & 49,9 \\
\hline Brustschmerz & 5-stufig & $2,62 \pm 0,03$ & $1,13 \pm 0,02$ & 1,49 & 57,1 \\
\hline Atemnot & 5-stufig & $2,57 \pm 0,03$ & $1,14 \pm 0,02$ & 1,43 & 55,5 \\
\hline Schlafstörungen & 5-stufig & $2,99 \pm 0,03$ & $1,25 \pm 0,02$ & 1,74 & 58,1 \\
\hline Abhusten von Schleim & 5-stufig & $3,13 \pm 0,03$ & $1,50 \pm 0,02$ & 1,63 & 52,1 \\
\hline Hustenattacken & 4-stufig & $2,76 \pm 0,02$ & $1,60 \pm 0,02$ & 1,16 & 42,1 \\
\hline
\end{tabular}




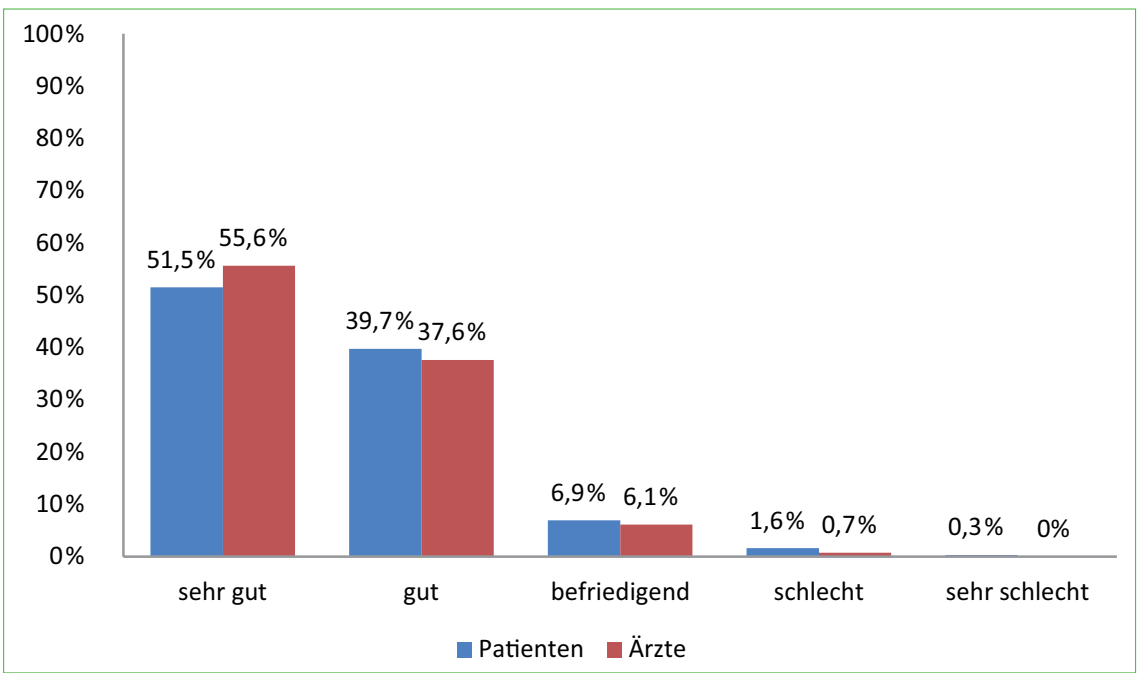

- Abb. 2 Abschließende Bewertung der Wirksamkeit durch die Ärzte und Patienten bzw. deren Eltern.

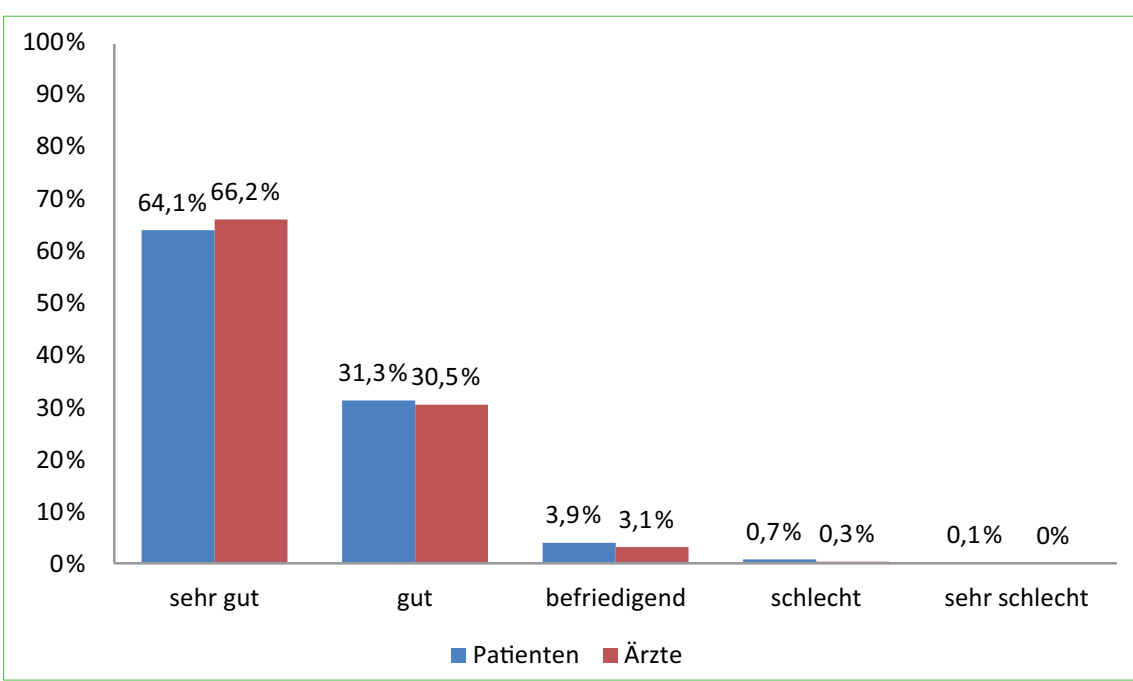

- Abb. 3 Abschließende Bewertung der Verträglichkeit durch die Ärzte und Patienten bzw. deren Eltern.

Mittel 1,60 Punkte, entsprechend etwa 5 Attacken pro Tag.

Auch bei diesen beiden Parametern konnte eine sehr gute Übereinstimmung mit den Daten aus dem Patiententagebuch gezeigt werden $(3,04 \pm 0,03$ auf 1,46 $\pm 0,02$ bzw. $2,84 \pm 0,03$ auf 1,66 $\pm 0,02$ ) . Zusätzlich wurden ausschließlich im Patiententagebuch der Schweregrad der allgemeinen Beschwerden und Symptome sowie die Häufigkeit des nächtlichen Aufwachens abgefragt. Hier kam es zu einer Reduktion von $2,99 \pm 0,03$ auf $1,40 \pm 0,02$ (53,2\%) bzw. 2,41 $\pm 0,03$ auf $1,23 \pm 0,02$ entsprechend einer rechnerischen Reduktion des nächtlichen Aufwachens von ca. 7- auf ca. 2-mal. men ihn 92,4\% der Patienten als angenehm oder sehr angenehm wahr. Zusätzlich bieten die 5 unterschiedlichen Darreichungsformen eine hohe Adaptionsmöglichkeit an die Bedürfnisse der Patienten.

\section{Beurteilung der globalen Wirksamkeit und Verträglichkeit}

Die Beurteilung der globalen Wirksamkeit und Verträglichkeit erfolgte im Rahmen der Abschlussvisite und wurde sowohl von den Ärzten als auch von den Patienten bzw. deren Eltern vorgenommen. Die Wirksamkeit der Therapie wurde von den Ärzten in 93,2\% der Fälle und von 91,2\% der Patienten mit „gut“ oder „sehr gut“ bewertet ( $\triangleright$ Abb. 2). Die Verträglichkeit schätzten die Ärzte in 96,7\% der Fälle und 95,4\% der Patienten mit „gut“ oder „sehr gut“ ein ( 10 Patienten $(0,94 \%)$ wurde eine UAW (7 gastrointestinale, 2 allergische, 1 andere) dokumentiert.

\section{Diskussion}

Die Ergebnisse dieser nicht-interventionellen Studie bestätigen die Wirksamkeit von Arzneimitteln mit dem Efeu-Spezialextrakt EA $575^{\circledR}$ und zwar in allen untersuchten Darreichungsformen bei Schulkindern mit akuter Bronchitis im Alter von 6-12 Jahren. Diese profitierten v.a. von der Verbesserung der Symptome „Atemnot“ und „Abhusten von Schleim“. Dies steht im Einklang mit dem nachgewiesenen Wirkmechanismus von einem wichtigen Inhaltsstoff dieses Efeu-Spezialextraktes: $\alpha$-Hederin hemmt die Internalisierung von $\beta_{2}$-Adrenorezeptoren, wirkt somit als indirektes $\beta_{2}$-Sympathomimetikum und führt dadurch zu einer erhöhten Bronchospasmolyse und Sekretolyse [13]. Dieser sekretolytische und bronchospasmolytische Effekt von EA $575^{\circledR}$ wurde bereits u.a. durch die Erhebung objektiver Lungenparameter durch Spirometrie und Bodyplethysmographie im Rahmen von klinischen Studien an Patienten mit akuten und chronischen Erkrankungen der Atemwege gezeigt [8]. Weiterhin erfuhren die Patienten eine deutliche Reduktion des Brustschmerzes beim Husten sowie eine sowohl für die Eltern der Patienten als auch für den Pati- 
enten selbst entlastende Reduktion der nächtlichen Schlafstörungen. Eine deutlich wahrnehmbare Verbesserung der Symptome zeigte sich bereits nach 3,48 Tagen, was sehr gut mit den Daten aus einer kontrollierten klinischen Studie an Asthma-Patienten übereinstimmt. Auch hier stellte sich der volle Effekt der EA $575^{\circledR}$-Therapie nach 3 Tagen ein [9].

Die Besserung der Gesamtsymptomatik zeigt sich in dieser NIS auch in einer deutlichen Reduktion des klinisch validierten Bronchitis Severity Score (BSS) um 79,3\%. Interessant ist hierbei die Tatsache, dass kein relativer Unterschied zwischen den Patienten mit und ohne Begleittherapie zu erkennen war. Dies legt nahe, dass in manchen Fällen eine Einzelverordnung der sehr gut verträglichen EA $575^{\circledR}$-haltigen Darreichungsformen verglichen mit einer Kombination z.B. mit Analgetika oder Antibiotika - bereits ausreichen kann. Trotz der Einnahme verschiedener Medikamente zur Behandlung von Begleiterkrankungen oder Begleitinfektionen wurden keine Wechselwirkungen beobachtet.

Der Therapieeffekt der ca. einwöchigen Behandlung wurde auch durch die hohe Therapiecompliance sichergestellt, die im angenehm empfundenen Geschmack sowie in der Verfügbarkeit 5 verschiedener Darreichungsformen ihre Ursache hat. Die Sicherheit und Verträglichkeit der Therapie zeigte sich in der niedrigen Inzidenz an UAW (0,94\%). Die erfolgreiche Therapie von Schulkindern mit akuter Bronchitis mit allen EA $575^{\circledR}$-Darreichungsformen wird durch die nahezu ausschließlich mit „gut“ oder „sehr gut“ bewertete globale Wirksamkeit und Verträglichkeit unterstrichen.

Interessenkonflikt: $\mathrm{CL}$ und $\mathrm{CS}$ sind Mitarbeiter der Engelhard Arzneimittel GmbH \& Co. KG. TW wurde mit der Planung, Auswertung und zum Teil mit der Durchführung der Studie von der Firma Engelhard Arzneimittel beauftragt.
Dr. Christopher Lang

Engelhard Arzneimittel GmbH \& Co. KG

Herzbergstr. 3

61138 Niederdorfelden

c.lang@engelhard.de

Online

http://dx.doi.org/10.1055/s-0041-105237

\section{Literatur}

1 Böhlau V. Therapeutische Erfahrungen mit Prospan bei chronisch-obstruktiven Atemwegserkrankungen. Notabene medici 1977: 26-29

2 DEGAM-Leitlinie Nr. 11. Husten. Düsseldorf: Omicron Publishing; 2008: 1-64

3 Doull IJ, Williams AA, Freezer NJ, Holgate ST. Descriptive study of cough, wheeze and school absence in childhood. Thorax 1996; 51: 630631

4 Empfehlungen des Bundesinstituts für Arzneimittel und Medizinprodukte und des Paul-Ehrlich-Instituts zur Planung, Durchführung und Auswertung von Anwendungsbeobachtungen. 7. Juli 2010. http://www.bfarm.de

5 Friede KH. Aerosol-Behandlung der Bronchitis mit Prospan. Med Klinik 1957; 52: 62-63

6 HMPC meeting report on Community herbal monographs, guidelines and other activities. EMA/HMPC/301544/2013

7 Kraft K, Loew D, Schneider B, Kemper FH. Planung, Durchführung und Auswertung von Anwendungsbeobachtungen. Empfehlungen der Gesellschaft für Phytotherapie (GPHY). Arzneim-Forsch/Drug Res 1997; 47: 990-994

8 Lang C, Röttger-Lüer P, Staiger C. A valuable option for the treatment of respiratory disea-

\section{Summary}

Ivy in everyday paediatric use: Administration of EA $575^{\circledR}$ to schoolchildren for the treatment of acute bronchitis

The efficacy and tolerability of five different administration forms containing the special ivy extract EA $575^{\circledR}$ were investigated in a non-interventional study involving over 1000 schoolchildren (aged 6 to 12) suffering from acute bronchitis. Efficacy was recorded by means of an assessment of the findings on the part of the physicians, an assessment of the patients involving questioning of their typical symptoms as well as by means of a diary. There was a clear improvement in all findings and symptoms over the course of the 7-day treatment. What is more, the administration forms proved to be very well tolerated and the patients exhibited a high level of compliance. All five investigated EA $575^{\circledR}$ administration forms thus constitute an effective and reliable form of treatment for acute bronchitis amongst the examined group of schoolchildren, and, consequently, confirm the many years of EA $575^{\circledR}$ use. 


\section{Schlüsselwörter}

Efeublätter-Trockenextrakt EA 575 ${ }^{\circledR}$, Pro-

$\operatorname{span}^{\circledR}$, Hedera helix L., nicht-interventionelle

Studie; Schulkinder, Wirksamkeit, Verträg-

lichkeit, Compliance

Key words

EA $575^{\circledR}$ dried ivy leaf extract, Hedera helix L., non-interventional study; schoolchildren,

efficacy, tolerability, compliance 